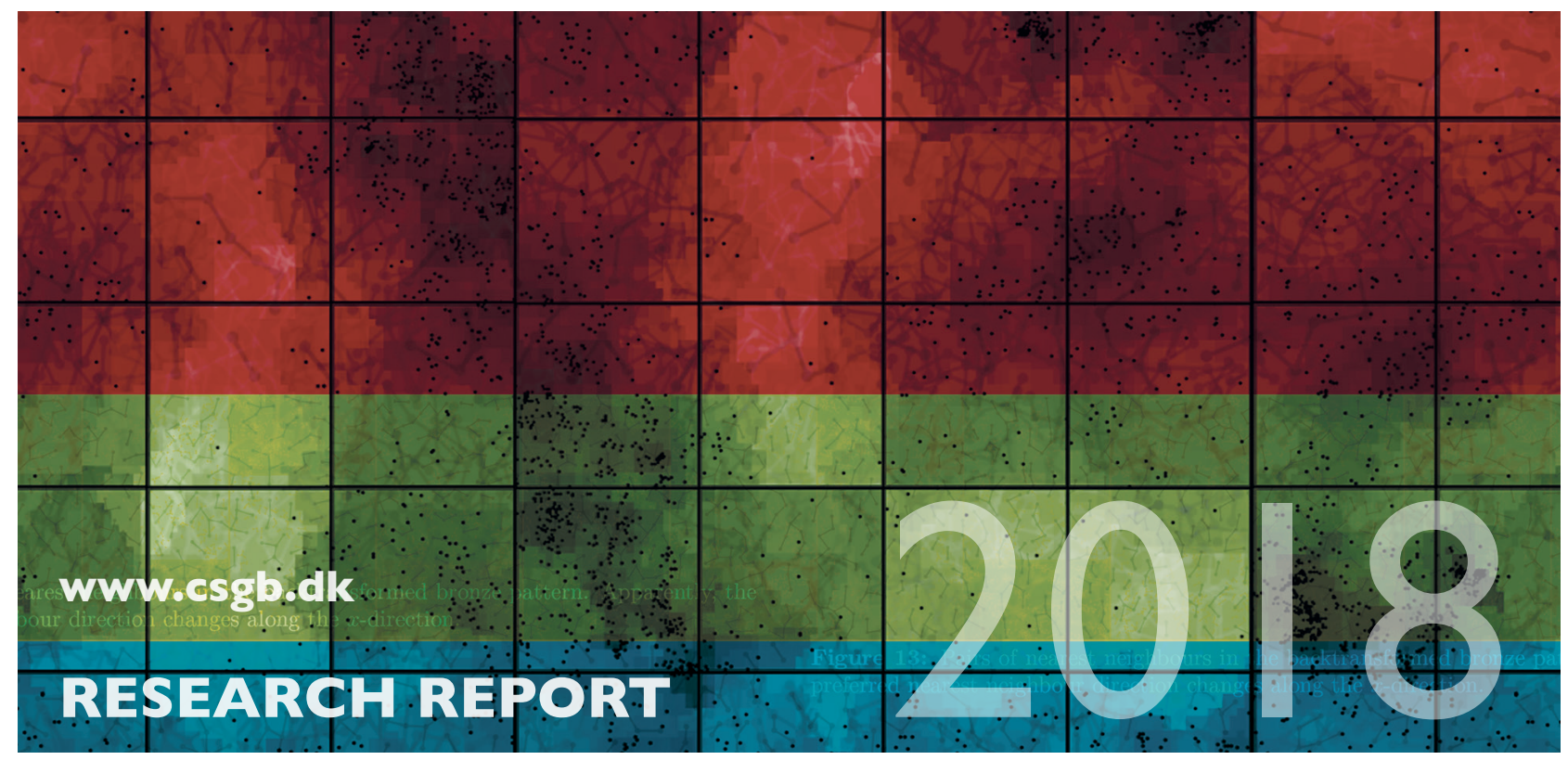

Anders Rønn-Nielsen and Eva B. Vedel Jensen

Central limit theorem for mean and variogram estimators in Lévy-based models

No. 06, June 2018 


\title{
Central limit theorem for mean and variogram estimators in Lévy-based models
}

\author{
Anders Rønn-Nielsen ${ }^{1}$ and Eva B. Vedel Jensen ${ }^{2}$ \\ ${ }^{1}$ Department of Finance, Copenhagen Business School \\ ${ }^{2}$ Department of Mathematics, Aarhus University
}

\begin{abstract}
We consider an infinitely divisible random field in $\mathbb{R}^{d}$ given as an integral of a kernel function with respect to a Lévy basis. Under mild regularity conditions we derive central limit theorems for the moment estimators of the mean and the variogram of the field.
\end{abstract}

Keywords: central limit theorem; infinite divisibility; Lévy-based modelling

\section{Introduction}

In the present paper, we derive central limit theorems (CLTs) for mean and variogram estimators for a field $\left(X_{i}\right)_{i \in \mathbb{Z}^{d}}$, defined by

$$
X_{i}=\int_{\mathbb{R}^{d}} f(s-i) M(\mathrm{~d} s),
$$

where $M$ is an infinitely divisible, independently scattered random measure on $\mathbb{R}^{d}$ and $f$ is some kernel function.

Lévy-based models as defined in (1.1) provide a flexible modelling framework that recently has been used in a range of modelling contexts, including modelling of turbulent flows, growth processes, Cox point processes and brain imaging data $[3,6,8,9]$.

Due to the tractability of Lévy-based models, it has been possible to derive tail asymptotics for the supremum of such a field as well as asymptotics of excursion sets of the field. The case where $M$ is a convolution equivalent measure is considered in $[12,14]$. Results for random measures with regularly varying tails have been derived in [11] and refined in [1, 2]. by

In [4], CLTs are proved for a stationary random field $\left(X_{i}\right)_{i \in \mathbb{Z}^{d}}$, discretely defined

$$
X_{i}=u\left(M_{s+i}: s \in \mathbb{Z}^{d}\right),
$$

where $\left(M_{i}\right)_{i \in \mathbb{Z}^{d}}$ are independent and identically distributed (i.i.d.) random variables and $u$ is a measurable function. In contrast, our set-up involves a random measure 
defined on $\mathbb{R}^{d}$. However, if we discretize the integral in (1.1), then our model is of the form (1.2) with $u$ a linear function and the random variables $M_{i}$ having an infinitely divisible distribution. Note that if this type of model is going to hold for finer and finer resolution, then the common distribution of the random variables $M_{i}$ has to be infinitely divisible.

For a random field defined by (1.1), we prove in the present paper CLTs for

$$
S_{\Gamma}=\sum_{i \in \Gamma} X_{i} \quad \text { and } \quad T_{\Gamma}(h)=\sum_{i \in \Gamma}\left(X_{i}-X_{i+h}\right)^{2},
$$

where $h \in \mathbb{Z}^{d}$ and $\Gamma$ is a finite subset of $\mathbb{Z}^{d}$ with number of elements increasing to infinity. We show under mild regularity conditions that both $S_{\Gamma}$ and $T_{\Gamma}(h)$ are asymptotically normally distributed and give explicit expressions for the asymptotic variances. It turns out that the asymptotic variance of $T_{\Gamma}(h)$ is equal to an approximate variance, earlier derived in [13]. In the latter paper, the approximate variance was used in the assessment of the precision of a section spacing estimator in electron microscopy. More details are given in Section 5 below.

If we discretize the integral in (1.1), both $S_{\Gamma}$ and $T_{\Gamma}(h)$ take the form

$$
\sum_{i \in \Gamma} v\left(\sum_{s \in \mathbb{Z}^{d}} a_{s} M_{s+i}\right) .
$$

Here, $v(x)=x$ and $a_{s}=f(s)$ for $S_{\Gamma}$, while $v(x)=x^{2}$ and $a_{s}=f(s)-f(s-h)$ for $T_{\Gamma}(h)$. In [4], CLTs for random variables of the form (1.3) are discussed. In particular, a CLT for $S_{\Gamma}$ is derived which is a discrete analogue of the CLT we obtain. For second-order properties, [4] considers

$$
\sum_{i \in \Gamma} X_{i} X_{i+h}
$$

but do not deal explicitly with the case where the mean of the field is unknown, as is done in our paper.

The present paper is organised as follows. In Section 2 we define the random field (1.1) and state the main result. In Section 3 we provide the proofs. An important tool in the proofs will be a CLT (Theorem 3.1) for $m_{n}$-dependent fields proved in [5]. Section 4 discusses the case where the kernel function is isotropic, while the results are used for estimation of sample spacing in Section 5. Cumulant formulae and a proof of a corollary to Ottaviani's inequality are deferred to two Appendices.

\section{Preliminaries and main result}

Consider an independently scattered random measure $M$ on $\mathbb{R}^{d}$. Then for a sequence of disjoint sets $\left(A_{n}\right)_{n \in \mathbb{N}} \subseteq \mathbb{R}^{d}$ in $\mathcal{B}\left(\mathbb{R}^{d}\right)$ the random variables $\left(M\left(A_{n}\right)\right)_{n \in \mathbb{N}}$ are independent and satisfy $M\left(\cup A_{n}\right)=\sum M\left(A_{n}\right)$. Assume furthermore that $M(A)$ is infinitely divisible for all $A \in \mathcal{B}\left(\mathbb{R}^{d}\right)$. Then $M$ is called a Lévy basis, see [3] and references therein. 
For a random variable $X$ let $\kappa_{X}(\lambda)$ denote its cumulant function $\log E\left(e^{i \lambda X}\right)$. We shall assume that the Lévy basis is stationary and isotropic such that for $A \in \mathcal{B}\left(\mathbb{R}^{d}\right)$ the variable $M(A)$ has a Lévy-Khintchine representation given by

$$
\kappa_{M(A)}(\lambda)=i \lambda a m_{d}(A)-\frac{1}{2} \lambda^{2} \theta m_{d}(A)+\int_{A \times \mathbb{R}}\left(e^{i \lambda u}-1-i \lambda u 1_{[-1,1]}(u)\right) F(\mathrm{~d} s, \mathrm{~d} u)
$$

where $m_{d}$ is the Lebesgue measure on $\left(\mathbb{R}^{d}, \mathcal{B}\left(\mathbb{R}^{d}\right)\right), a \in \mathbb{R}, \theta \geq 0$ and $F$ is a measure on $\mathcal{B}\left(\mathbb{R}^{d} \times \mathbb{R}\right)$ of the form

$$
F(A \times B)=m_{d}(A) \rho(B) .
$$

In the following, we let $M^{\prime}$ be a so-called spot variable, i.e. $M^{\prime}$ is a random variable with the distribution of $M(A)$ for $A \in \mathcal{B}\left(\mathbb{R}^{d}\right)$ with $m_{d}(A)=1$. We will assume that the Lévy measure $\rho$ satisfies

$$
\int_{[-1,1]} z^{2} \rho(\mathrm{d} z)<\infty \text { and } \int_{[-1,1]^{c}}|z| \rho(\mathrm{d} z)<\infty .
$$

The first assumption is needed for $\rho$ to be a Lévy measure. The second assumption is equivalent to $E\left|M^{\prime}\right|<\infty$ (see [15, Theorem 25.3]). Now assume that $f: \mathbb{R}^{d} \rightarrow[0, \infty)$ is a bounded kernel function satisfying

$$
\int_{\mathbb{R}^{d}} f(s) \mathrm{d} s<\infty
$$

Let $\mathbb{Z}^{d}$ be the grid integer numbers in $\mathbb{R}^{d}$. We consider the family of random variables $\left(X_{i}\right)_{i \in \mathbb{Z}^{d}}$ defined by

$$
X_{i}=\int_{\mathbb{R}^{d}} f(s-i) M(\mathrm{~d} s) .
$$

See [10, Theorem 2.7] for existence of the integrals, where their conditions (i)(iii) can be easily verified under the given assumptions on $M$ and $f$. The field is stationary, and if furthermore $f$ has the form $f(s)=g(\|s\|)$, where $\|\cdot\|$ is Euclidean norm, it will be isotropic. This special case is studied in Section 4.

We will be interested in estimating the mean and the variogram of the field. We have

$$
\mu=\mathbb{E} X_{0}=\mathbb{E}\left(M^{\prime}\right) \int_{\mathbb{R}^{d}} f(s) \mathrm{d} s
$$

and for $h \in \mathbb{Z}^{d}$

$$
\gamma(h)=\mathbb{E}\left(X_{0}-X_{h}\right)^{2}=\operatorname{Var}\left(M^{\prime}\right) \int_{\mathbb{R}^{d}}(f(s)-f(s-h))^{2} \mathrm{~d} s,
$$

where identities from Appendix A have been applied. Note that due to stationarity, $\mu=\mathbb{E} X_{i}$ and $\gamma(h)=\mathbb{E}\left(X_{i}-X_{i+h}\right)^{2}$ for all $i \in \mathbb{Z}^{d}$. For $\Gamma$ a finite subset of $\mathbb{Z}^{d}$, we define

$$
S_{\Gamma}=\sum_{i \in \Gamma} X_{i}
$$


and for $h \in \mathbb{Z}^{d}$

$$
T_{\Gamma}(h)=\sum_{i \in \Gamma}\left(X_{i}-X_{i+h}\right)^{2} .
$$

Unbiased estimators for $\mu$ and $\gamma(h)$, respectively, are obtained as

$$
\widehat{\mu}=\frac{1}{|\Gamma|} S_{\Gamma} \quad \widehat{\gamma(h)}=\frac{1}{|\Gamma|} T_{\Gamma}
$$

where $|\Gamma|$ denotes the number of elements in $\Gamma$. The main result gives asymptotic normality of these estimators for $|\Gamma|$ going to infinity.

Theorem 2.1. Let $\left(\Gamma_{n}\right)_{n \geq 1}$ be a sequence of finite subsets of $\mathbb{Z}^{d}$ and assume that $\left|\Gamma_{n}\right|$ goes to infinity and $\left|\partial \Gamma_{n}\right| /\left|\Gamma_{n}\right|$ goes to 0 . Let

$$
\sigma_{S}^{2}=\operatorname{Var}\left(M^{\prime}\right) \sum_{i \in \mathbb{Z}^{d}} \int_{\mathbb{R}^{d}} f(s) f(s-i) \mathrm{d} s .
$$

If $\sigma_{S}^{2}<\infty$, then

$$
\frac{S_{\Gamma_{n}}-\left|\Gamma_{n}\right| \mu}{\sqrt{\left|\Gamma_{n}\right|}} \stackrel{\mathcal{D}}{\rightarrow} \mathcal{N}\left(0, \sigma_{S}^{2}\right) .
$$

If furthermore $\mathbb{E}\left(M^{\prime}\right)^{4}<\infty$, then

$$
\frac{T_{\Gamma_{n}}(h)-\left|\Gamma_{n}\right| \gamma(h)}{\sqrt{\left|\Gamma_{n}\right|}} \stackrel{\mathcal{D}}{\rightarrow} \mathcal{N}\left(0, \sigma_{T}^{2}\right),
$$

where

$\sigma_{T}^{2}=\sum_{j \in \mathbb{Z}^{d}}\left[\kappa_{4}\left(M^{\prime}\right) \int_{\mathbb{R}^{d}} g(s)^{2} g(s-j)^{2} \mathrm{~d} s+2 \operatorname{Var}\left(M^{\prime}\right)^{2}\left(\int_{\mathbb{R}^{d}} g(s) g(s-j) \mathrm{d} s\right)^{2}\right]<\infty$, $\kappa_{4}\left(M^{\prime}\right)$ is the fourth cumulant of $M^{\prime}$ and $g(s)=f(s)-f(s-h)$.

It should be noted that the assumptions of the theorem

$$
\left|\Gamma_{n}\right| \rightarrow \infty \text { and }\left|\partial \Gamma_{n}\right| /\left|\Gamma_{n}\right| \rightarrow 0
$$

are equivalent with the (less intuitive, but more computationally convenient) property

$$
\left|\Gamma_{n}\right| \rightarrow \infty \quad \text { and } \quad\left|\left(\Gamma_{n}-j\right) \cap \Gamma_{n}\right| /\left|\Gamma_{n}\right| \rightarrow 1
$$

for all $j \in \mathbb{Z}^{d}$, where $\Gamma+j$ denotes translation of $\Gamma$ in direction $j \in \mathbb{Z}^{d}$, i.e.

$$
\Gamma+j=\{i+j \mid i \in \Gamma\}
$$




\section{Proofs}

An important tool in the proof of our main result, Theorem 2.1, is a CLT for $m_{n}-$ dependent random fields that may be found in [5, Theorem 2]. This theorem is also used in the proofs of the CLTs, derived in [4]. However, our proof of the CLT for $S_{\Gamma}$ is simpler and takes advantage of the linearity of the Lévy-based model (1.1), while our proof of the CLT for $T_{\Gamma}(h)$ follows a different route than the one adopted in [4] for the second-order property $\sum_{i \in \Gamma} X_{i} X_{i+h}$.

Recall that a random field $\left(Z_{i}\right)_{i \in \mathbb{Z}^{d}}$ is said to be $m_{n}$-dependent, if for any $A, B \subset$ $\mathbb{Z}^{d}$ with $|A|,|B|<\infty$ and $\inf \{\|a-b\|: a \in A, b \in B\}>m_{n},\left(Z_{a}\right)_{a \in A}$ and $\left(Z_{b}\right)_{b \in B}$ are independent. The CLT for $m_{n}$-dependent fields is here stated as

Theorem 3.1. Let $\left(\Gamma_{n}\right)_{n \geq 1}$ be a sequence of finite subsets of $\mathbb{Z}^{d}$ with $\left|\Gamma_{n}\right| \rightarrow \infty$ as $n \rightarrow \infty$ and let $\left(m_{n}\right)_{n \geq 1}$ be a sequence of positive integers. For each $n \geq 1$, let $\left\{U_{n}(j), j \in \mathbb{Z}^{d}\right\}$ be an $m_{n}$-dependent random field with $\mathbb{E} U_{n}(j)=0$ for all $j \in \mathbb{Z}^{d}$. Assume that $\mathbb{E}\left(\sum_{j \in \Gamma_{n}} U_{n}(j)\right)^{2} \rightarrow \sigma^{2}$ as $n \rightarrow \infty$ with $\sigma^{2}<\infty$. Then $\sum_{j \in \Gamma_{n}} U_{n}(j)$ converges in distribution to a Gaussian random variable with mean zero and variance $\sigma^{2}$, if there exists a finite constant $c>0$ such that for any $n \geq 1$,

$$
\sum_{j \in \Gamma_{n}} \mathbb{E} U_{n}(j)^{2} \leq c
$$

and for any $\epsilon>0$, it holds that $\lim _{n \rightarrow \infty} L_{n}(\epsilon)=0$, where

$$
L_{n}(\epsilon)=m_{n}^{2 d} \sum_{j \in \Gamma_{n}} \mathbb{E}\left(U_{n}(j)^{2} \mathbf{1}_{\left\{\left|U_{n}(j)\right| \geq \epsilon m_{n}^{-2 d}\right\}}\right) .
$$

Proof of Theorem 2.1 for $S_{\Gamma_{n}}$. Let $\left(m_{n}\right)_{n \geq 1}$ be a sequence of integers increasing to infinity. The choice of the sequence will be specified below.

Let for $i \in \mathbb{Z}^{d}$ and $n \in \mathbb{N}, C_{n}(i)$ be the ball in $\mathbb{R}^{d}$ with center in $i$ and radius $m_{n} / 3$. Let

$$
\bar{X}_{i}^{n}=\int_{C_{n}(i)} f(s-i) M(\mathrm{~d} s)
$$

and let furthermore $\mu_{n}=\mathbb{E}\left(\bar{X}_{0}^{n}\right)=\mathbb{E}\left(M^{\prime}\right) \int_{C_{n}(0)} f(s) \mathrm{d} s$ and $\bar{S}_{\Gamma_{n}}=\sum_{i \in \Gamma_{n}} \bar{X}_{i}^{n}$. The proof will be divided into two steps. The first step is to show that

$$
\lim _{n \rightarrow \infty} \frac{\left\|S_{\Gamma_{n}}-\left|\Gamma_{n}\right| \mu-\left(\bar{S}_{\Gamma_{n}}-\left|\Gamma_{n}\right| \mu_{n}\right)\right\|_{2}}{\sqrt{\left|\Gamma_{n}\right|}}=0 .
$$

The second step is to show, using Theorem 3.1, that with an appropriately chosen sequence $\left(m_{n}\right)_{n \geq 1}$, we have

$$
\frac{\bar{S}_{\Gamma_{n}}-\left|\Gamma_{n}\right| \mu_{n}}{\sqrt{\left|\Gamma_{n}\right|}} \stackrel{\mathcal{D}}{\rightarrow} \mathcal{N}\left(0, \sigma_{S}^{2}\right)
$$


To prove (3.1), we find, using Appendix A,

$$
\begin{aligned}
& \frac{\left\|S_{\Gamma_{n}}-\left|\Gamma_{n}\right| \mu-\left(\bar{S}_{\Gamma_{n}}-\left|\Gamma_{n}\right| \mu_{n}\right)\right\|_{2}^{2}}{\left|\Gamma_{n}\right|} \\
& =\frac{1}{\left|\Gamma_{n}\right|} \mathbb{E}\left[\left(\sum_{i \in \Gamma_{n}}\left[\int_{C_{n}(i)^{c}} f(s-i) M(\mathrm{~d} s)-\mathbb{E}\left(M^{\prime}\right) \int_{C_{n}(i)^{c}} f(s-i) \mathrm{d} s\right]\right)^{2}\right] \\
& =\frac{1}{\left|\Gamma_{n}\right|} \sum_{i \in \Gamma_{n}} \sum_{j \in \Gamma_{n}} \operatorname{Cov}\left[\int_{C_{n}(i)^{c}} f(s-i) M(\mathrm{~d} s), \int_{C_{n}(j)^{c}} f(s-j) M(\mathrm{~d} s)\right] \\
& =\frac{\operatorname{Var}\left(M^{\prime}\right)}{\left|\Gamma_{n}\right|} \sum_{i \in \Gamma_{n}} \sum_{j \in \Gamma_{n}} \int_{C_{n}(i)^{c} \cap C_{n}(j)^{c}} f(s-i) f(s-j) \mathrm{d} s \\
& \leq \frac{\operatorname{Var}\left(M^{\prime}\right)}{\left|\Gamma_{n}\right|} \sum_{i \in \Gamma_{n}} \sum_{j \in \mathbb{Z}^{d}} \int_{C_{n}(i)^{c} \cap C_{n}(j)^{c}} f(s-i) f(s-j) \mathrm{d} s \\
& =\operatorname{Var}\left(M^{\prime}\right) \sum_{j \in \mathbb{Z}^{d}} \int_{C_{n}(0)^{c} \cap C_{n}(j)^{c}} f(s) f(s-j) \mathrm{d} s \\
& \rightarrow 0 \text {, }
\end{aligned}
$$

where the convergence is a result of dominated convergence, since

$$
\sum_{j \in \mathbb{Z}^{d}} \int_{\mathbb{R}^{d}} f(s) f(s-j) \mathrm{d} s<\infty,
$$

and $C_{n}(0)^{c} \cap C_{n}(j)^{c} \rightarrow \emptyset$ as $n \rightarrow \infty$.

In order to prove (3.2), define for $n \in \mathbb{N}$ and $j \in \mathbb{Z}^{d}$

$$
U_{n}(j)=\frac{\bar{X}_{j}^{n}-\mu_{n}}{\sqrt{\left|\Gamma_{n}\right|}},
$$

such that

$$
\sum_{j \in \Gamma_{n}} U_{n}(j)=\frac{\bar{S}_{\Gamma_{n}}-\left|\Gamma_{n}\right| \mu_{n}}{\sqrt{\left|\Gamma_{n}\right|}} .
$$

Then, clearly $\left(U_{n}(j)\right)_{j \in \mathbb{Z}^{d}}$ is $m_{n}$-dependent with $\mathbb{E} U_{n}(j)=0$ for all $n \geq 1$ and $j \in \mathbb{Z}^{d}$. Furthermore, cf. Appendix A,

$$
\begin{aligned}
& \mathbb{E}\left[\left(\sum_{i \in \Gamma_{n}} U_{n}(i)\right)^{2}\right] \\
& \quad=\frac{1}{\left|\Gamma_{n}\right|} \sum_{i \in \Gamma_{n}} \sum_{j \in \Gamma_{n}} \operatorname{Cov}\left[\int_{C_{n}(i)} f(s-i) M(\mathrm{~d} s), \int_{C_{n}(j)} f(s-j) M(\mathrm{~d} s)\right] \\
& =\frac{\operatorname{Var}\left(M^{\prime}\right)}{\left|\Gamma_{n}\right|} \sum_{i \in \Gamma_{n}} \sum_{j \in \Gamma_{n}} \int_{C_{n}(i) \cap C_{n}(j)} f(s-i) f(s-j) \mathrm{d} s \\
& =\operatorname{Var}\left(M^{\prime}\right) \sum_{j \in \mathbb{Z}^{d}} \frac{\left|\left(\Gamma_{n}-j\right) \cap \Gamma_{n}\right|}{\left|\Gamma_{n}\right|} \int_{C_{n}(0) \cap C_{n}(j)} f(s) f(s-j) \mathrm{d} s \\
& \quad \rightarrow \operatorname{Var}\left(M^{\prime}\right) \sum_{j \in \mathbb{Z}^{d}} \int_{\mathbb{R}^{d}} f(s) f(s-j) \mathrm{d} s,
\end{aligned}
$$


as $n \rightarrow \infty$, by dominated convergence. Here, we have used that (3.3) is fulfilled, $\left|\left(\Gamma_{n}-j\right) \cap \Gamma_{n}\right| /\left|\Gamma_{n}\right| \rightarrow 1$ for all $j$ and $C_{n}(0) \cap C_{n}(j) \rightarrow \mathbb{R}^{d}$ as $n \rightarrow \infty$. Furthermore, we find, cf. Appendix A,

$$
\sum_{i \in \Gamma_{n}} \mathbb{E}\left[U_{n}(i)^{2}\right]=\operatorname{Var}\left(\bar{X}_{0}^{n}\right)=\operatorname{Var}\left(M^{\prime}\right) \int_{C_{n}(0)} f(s)^{2} \mathrm{~d} s \leq \operatorname{Var}\left(M^{\prime}\right) \int_{\mathbb{R}^{d}} f(s)^{2} \mathrm{~d} s<\infty .
$$

Now define

$$
Y=\sup _{n \geq 1}\left|\bar{X}_{0}^{n}-\mu_{n}\right|
$$

Note that the sequence $\left(\bar{X}_{0}^{n}\right)_{n \geq 1}$ has independent increments and that

$$
\sup _{n \geq 1} \mathbb{E}\left[\left(\bar{X}_{0}^{n}-\mu_{n}\right)^{2}\right]=\sup _{n \geq 1} \operatorname{Var}\left(M^{\prime}\right) \int_{C_{n}(0)} f(s)^{2} \mathrm{~d} s \leq \operatorname{Var}\left(M^{\prime}\right) \int_{\mathbb{R}^{d}} f(s)^{2} \mathrm{~d} s<\infty .
$$

Then, $\mathbb{E} Y^{2}<\infty$, due to Corollary B.2. Furthermore,

$$
\begin{aligned}
L_{n}(\epsilon) & =m_{n}^{2 d} \sum_{j \in \Gamma_{n}} \mathbb{E}\left[U_{n}^{2}(j) \mathbf{1}_{\left\{\left|U_{n}(j)\right| \geq \epsilon m_{n}^{-2 d}\right\}}\right] \\
& =m_{n}^{2 d} \mathbb{E}\left[\left(\bar{X}_{0}^{n}-\mu_{n}\right)^{2} \mathbf{1}_{\left\{\left|\bar{X}_{0}^{n}-\mu_{n}\right| \geq \epsilon \sqrt{\left|\Gamma_{n}\right|} m_{n}^{-2 d}\right\}}\right] \\
& \leq m_{n}^{2 d} \mathbb{E}\left[Y^{2} \mathbf{1}_{\left\{Y \geq \epsilon \sqrt{\left|\Gamma_{n}\right|} m_{n}^{-2 d}\right\}}\right] \\
& =m_{n}^{2 d} \mathbb{E}\left[Y^{2} \mathbf{1}_{\left\{Y \geq \epsilon \sqrt{\left|\Gamma_{n}\right|} m_{n}^{-2 d}, Y \leq\left|\Gamma_{n}\right|^{1 / 4}\right\}}\right]+m_{n}^{2 d} \mathbb{E}\left[Y^{2} \mathbf{1}_{\left\{Y \geq \epsilon \sqrt{\left|\Gamma_{n}\right|} m_{n}^{-2 d}, Y>\left|\Gamma_{n}\right|^{1 / 4}\right\}}\right] \\
& \leq m_{n}^{2 d} \sqrt{\left|\Gamma_{n}\right|} \mid \mathbb{P}\left[Y \geq \epsilon \sqrt{\left|\Gamma_{n}\right|} m_{n}^{-2 d}\right]+m_{n}^{2 d} \mathbb{E}\left[Y^{2} \mathbf{1}_{\left\{Y>\left|\Gamma_{n}\right|^{1 / 4}\right\}}\right] \\
& \leq \frac{m_{n}^{6 d}}{\epsilon^{2} \sqrt{\left|\Gamma_{n}\right|}} \mathbb{E} Y^{2}+m_{n}^{2 d} \psi\left(\left|\Gamma_{n}\right|^{1 / 4}\right),
\end{aligned}
$$

where $\psi(x)=\mathbb{E}\left(Y^{2} 1_{\{Y \geq x\}}\right)$. Note that $\psi(x) \rightarrow 0$ as $x \rightarrow \infty$. With [.] denoting the integer part, now choose the sequence $\left(m_{n}\right)_{n \geq 1}$ as $m_{n}=\left[\left|\Gamma_{n}\right|^{\frac{1}{24 d}}\right]$ if $\psi\left(\left|\Gamma_{n}\right|^{1 / 4}\right)=0$ and

$$
m_{n}=\min \left\{\left[\psi\left(\left|\Gamma_{n}\right|^{1 / 4}\right)^{-\frac{1}{4 d}}\right],\left[\left|\Gamma_{n}\right|^{\frac{1}{24 d}}\right]\right\},
$$

otherwise. With this choice it is seen that $L_{n}(\epsilon) \rightarrow 0$ as $n \rightarrow \infty$. Thus we have from Theorem 3.1 that

$$
\frac{\bar{S}_{\Gamma_{n}}-\left|\Gamma_{n}\right| \mu_{n}}{\sqrt{\left|\Gamma_{n}\right|}} \stackrel{\mathcal{D}}{\rightarrow} \mathcal{N}\left(0, \sigma_{S}^{2}\right) .
$$

Combining this with (3.1) yields the desired result.

In the proof of Theorem 2.1 for $T_{\Gamma_{n}}(h)$, we need the following lemma, the proof of which follows from straightforward calculations, using that $f$ is bounded and non-negative.

Lemma 3.2. Let for $h \in \mathbb{Z}^{d}$

$$
g(s)=f(s)-f(s-h), \quad s \in \mathbb{R}^{d} .
$$


Then, for $s \in \mathbb{R}^{d}, j \in \mathbb{Z}^{d}$,

$$
\begin{gathered}
|g(s) g(s-j)| \leq v_{j}(s), \\
g(s)^{2} g(s-j)^{2} \leq C v_{j}(s),
\end{gathered}
$$

where

$v_{j}(s)=f(s) f(s-j)+f(s) f(s-h-j)+f(s-h) f(s-j)+f(s-h) f(s-h-j)$

and $C<\infty$ is an appropriately chosen constant. If

$$
\sum_{j \in \mathbb{Z}^{d}} \int_{\mathbb{R}^{d}} f(s) f(s-j) \mathrm{d} s<\infty
$$

then

$$
\sum_{j \in \mathbb{Z}^{d}} \int_{\mathbb{R}^{d}} v_{j}(s) \mathrm{d} s<\infty \quad \text { and } \sum_{j \in \mathbb{Z}^{d}}\left(\int_{\mathbb{R}^{d}} v_{j}(s) \mathrm{d} s\right)^{2}<\infty .
$$

Proof of Theorem 2.1 for $T_{\Gamma_{n}}(h)$. First note that it follows from Lemma 3.2 that $\sigma_{S}^{2}<\infty$ and $\mathbb{E}\left[\left(M^{\prime}\right)^{4}\right]<\infty$ implies $\sigma_{T}^{2}<\infty$.

For ease of notation, we shall now consider the field $\left(Y_{i}\right)_{i \in \mathbb{Z}^{d}}$, where $Y_{i}=X_{i}-X_{i+h}$. Then, for all $i \in \mathbb{Z}^{d}, \mathbb{E} Y_{i}=0$ and

$$
Y_{i}=\int_{\mathbb{R}^{d}}(f(s-i)-f(s-i-h)) M(\mathrm{~d} s)=\int_{\mathbb{R}^{d}} g(s-i) M(\mathrm{~d} s),
$$

where $g(s)=f(s)-f(s-h)$ as previously. Let $\left(m_{n}\right)_{n \geq 1}$ be (another) sequence of integers increasing to infinity. Let $C_{n}(i)$ and $\bar{X}_{i}^{n}$ be defined as in the proof of Theorem 2.1 for $S_{\Gamma_{n}}$. Let

$$
\bar{Y}_{i}^{n}=\bar{X}_{i}^{n}-\bar{X}_{i+h}^{n}=\int_{\mathbb{R}^{d}} g_{n}(s-i) M(\mathrm{~d} s),
$$

where $g_{n}(s)=\mathbf{1}_{C_{n}(0)}(s) f(s)-\mathbf{1}_{C_{n}(h)}(s) f(s-h)$. Then $g_{n}(s) \rightarrow g(s)$ for all $s \in \mathbb{R}^{d}$. Note that $\mathbb{E} \bar{Y}_{i}^{n}=0$ and let furthermore $\gamma(h)_{n}=\mathbb{E}\left(\bar{Y}_{0}^{n}\right)^{2}$. Also define $\bar{T}_{\Gamma_{n}}(h)=$ $\sum_{i \in \Gamma_{n}}\left(\bar{Y}_{i}^{n}\right)^{2}$.

As for $S_{\Gamma_{n}}$, the first step will be to show that

$$
\lim _{n \rightarrow \infty} \frac{\left\|T_{\Gamma_{n}}(h)-\left|\Gamma_{n}\right| \gamma(h)-\left(\bar{T}_{\Gamma_{n}}(h)-\left|\Gamma_{n}\right| \gamma(h)_{n}\right)\right\|_{2}}{\sqrt{\left|\Gamma_{n}\right|}}=0 .
$$

The second step is to show, using Theorem 3.1, that with an appropriately chosen sequence $\left(m_{n}\right)_{n \geq 1}$, we have

$$
\frac{\bar{T}_{\Gamma_{n}}(h)-\left|\Gamma_{n}\right| \gamma(h)_{n}}{\sqrt{\left|\Gamma_{n}\right|}} \stackrel{\mathcal{D}}{\rightarrow} \mathcal{N}\left(0, \sigma_{T}^{2}\right) .
$$


To prove (3.4), we find

$$
\begin{aligned}
\frac{\left\|T_{\Gamma_{n}}(h)-\left|\Gamma_{n}\right| \gamma(h)-\left(\bar{T}_{\Gamma_{n}}(h)-\left|\Gamma_{n}\right| \gamma(h)_{n}\right)\right\|_{2}^{2}}{\left|\Gamma_{n}\right|} \\
=\frac{1}{\left|\Gamma_{n}\right|} \mathbb{E}\left[\left(\sum_{i \in \Gamma_{n}}\left[Y_{i}^{2}-\mathbb{E} Y_{i}^{2}-\left(\left(\bar{Y}_{i}^{n}\right)^{2}-\mathbb{E}\left(\bar{Y}_{i}^{n}\right)^{2}\right)\right]\right)^{2}\right] \\
=\frac{1}{\left|\Gamma_{n}\right|} \sum_{i, j \in \Gamma_{n}} \mathbb{E}\left[\left(Y_{i}^{2}-\mathbb{E} Y_{i}^{2}\right)\left(Y_{j}^{2}-\mathbb{E} Y_{j}^{2}\right)\right] \\
\quad+\frac{1}{\left|\Gamma_{n}\right|} \sum_{i, j \in \Gamma_{n}} \mathbb{E}\left[\left(\left(\bar{Y}_{i}^{n}\right)^{2}-\mathbb{E}\left(\bar{Y}_{i}^{n}\right)^{2}\right)\left(\left(\bar{Y}_{j}^{n}\right)^{2}-\mathbb{E}\left(\bar{Y}_{j}^{n}\right)^{2}\right)\right] \\
\quad-2 \frac{1}{\left|\Gamma_{n}\right|} \sum_{i, j \in \Gamma_{n}} \mathbb{E}\left[\left(Y_{i}^{2}-\mathbb{E} Y_{i}^{2}\right)\left(\left(\bar{Y}_{j}^{n}\right)^{2}-\mathbb{E}\left(\bar{Y}_{j}^{n}\right)^{2}\right)\right] .
\end{aligned}
$$

We will show convergence of the three terms in (3.6) separately. Using the calculation formulae from Appendix A, the first term equals

$$
\begin{aligned}
\frac{1}{\left|\Gamma_{n}\right|} \sum_{i, j \in \Gamma_{n}}\left[\kappa_{4}\left(M^{\prime}\right) \int_{\mathbb{R}^{d}} g(s-i)^{2} g(s-j)^{2} \mathrm{~d} s\right. & \\
& \left.+2 \operatorname{Var}\left(M^{\prime}\right)^{2}\left(\int_{\mathbb{R}^{d}} g(s-i) g(s-j) \mathrm{d} s\right)^{2}\right] \\
= & \sum_{j \in \mathbb{Z}^{d}} \frac{\left|\Gamma_{n} \cap\left(\Gamma_{n}-j\right)\right|}{\left|\Gamma_{n}\right|}\left[\kappa_{4}\left(M^{\prime}\right) \int_{\mathbb{R}^{d}} g(s)^{2} g(s-j)^{2} \mathrm{~d} s\right. \\
& \left.+2 \operatorname{Var}\left(M^{\prime}\right)^{2}\left(\int_{\mathbb{R}^{d}} g(s) g(s-j) \mathrm{d} s\right)^{2}\right] .
\end{aligned}
$$

By dominated convergence, this converges to $\sigma_{T}^{2}$, using that $\sigma_{T}^{2}$ is finite and that all terms in $\sigma_{T}^{2}$ are non-negative. The second term in (3.6) equals

$$
\begin{aligned}
\sum_{j \in \mathbb{Z}^{d}} \frac{\left|\Gamma_{n} \cap\left(\Gamma_{n}-j\right)\right|}{\left|\Gamma_{n}\right|}[ & \kappa_{4}\left(M^{\prime}\right) \int_{\mathbb{R}^{d}} g_{n}(s)^{2} g_{n}(s-j)^{2} \mathrm{~d} s \\
& \left.+2 \operatorname{Var}\left(M^{\prime}\right)^{2}\left(\int_{\mathbb{R}^{d}} g_{n}(s) g_{n}(s-j) \mathrm{d} s\right)^{2}\right] .
\end{aligned}
$$

By dominated convergence and Lemma 3.2, applied to $g_{n}$ instead of $g$, but keeping the upper bound as $v_{j}(s)$, this also converges to $\sigma_{T}^{2}$. Similarly, the third term of (3.6) converges to $-2 \sigma_{T}^{2}$, and we have the desired result in (3.4).

Now define for $n \in \mathbb{N}$ and $j \in \mathbb{Z}^{d}$

$$
V_{n}(j)=\frac{\left(\bar{Y}_{j}^{n}\right)^{2}-\gamma(h)_{n}}{\sqrt{\left|\Gamma_{n}\right|}} .
$$

We want to show that

$$
\sum_{j \in \Gamma_{n}} V_{n}(j) \stackrel{\mathcal{D}}{\rightarrow} \mathcal{N}\left(0, \sigma_{T}^{2}\right),
$$


using Theorem 3.1. Then by construction $\mathbb{E} V_{n}(j)=0$ for all $n \geq 1$ and $j \in \mathbb{Z}^{d}$, and for $n$ such that $m_{n} / 3>\|h\|$, the field $\left(V_{n}(j)\right)_{j \in \mathbb{Z}^{d}}$ is $m_{n}$-dependent. Furthermore

$$
\begin{aligned}
\mathbb{E}\left[\left(\sum_{i \in \Gamma_{n}} V_{n}(i)\right)^{2}\right] & =\frac{1}{\left|\Gamma_{n}\right|} \mathbb{E}\left[\left(\sum_{i \in \Gamma_{n}}\left[\left(\bar{Y}_{i}^{n}\right)^{2}-\mathbb{E}\left(\bar{Y}_{i}^{n}\right)^{2}\right]\right)^{2}\right] \\
& \left.=\frac{1}{\left|\Gamma_{n}\right|} \sum_{i, j \in \Gamma_{n}} \mathbb{E}\left[\left(\bar{Y}_{i}^{n}\right)^{2}-\mathbb{E}\left(\bar{Y}_{i}^{n}\right)^{2}\right)\left(\left(\bar{Y}_{j}^{n}\right)^{2}-\mathbb{E}\left(\bar{Y}_{j}^{n}\right)^{2}\right)\right]
\end{aligned}
$$

which equals the second term of (3.6) and therefore has limit $\sigma_{T}^{2}$. Additionally, using Appendix A,

$$
\begin{array}{rl}
\sum_{i \in \Gamma_{n}} & \mathbb{E} \\
& {\left[V_{n}(i)^{2}\right]=\mathbb{E}\left[\left[\left(\bar{Y}_{0}^{n}\right)^{2}-\mathbb{E}\left(\bar{Y}_{0}^{n}\right)^{2}\right]^{2}\right]} \\
& =\kappa_{4}\left(M^{\prime}\right)\left(\int_{\mathbb{R}^{d}} g_{n}(s)^{4} \mathrm{~d} s\right)+2 \operatorname{Var}\left(M^{\prime}\right)^{2}\left(\int_{\mathbb{R}^{d}} g_{n}(s)^{2} \mathrm{~d} s\right)^{2} \\
& \leq \kappa_{4}\left(M^{\prime}\right)\left(\int_{\mathbb{R}^{d}} v_{0}(s)^{2} \mathrm{~d} s\right)+2 \operatorname{Var}\left(M^{\prime}\right)^{2}\left(\int_{\mathbb{R}^{d}} v_{0}(s) \mathrm{d} s\right)^{2}<\infty
\end{array}
$$

with $v_{0}$ as defined in Lemma 3.2. Now define

$$
Z=\left(\sup _{n \geq 1}\left|\bar{X}_{0}^{n}\right|+\sup _{n \geq 1}\left|\bar{X}_{h}^{n}\right|\right)^{2}+C
$$

where

$$
C=2 \operatorname{Var}\left(M^{\prime}\right) \int_{\mathbb{R}^{d}} f(s)^{2} \mathrm{~d} s .
$$

Using Appendix A, we find that $0 \leq \gamma(h)_{n} \leq C$ for all $n \geq 1$. Also note that the sequence $\left(\bar{X}_{0}^{n}\right)_{n \geq 1}$ has independent increments and that for $k=1,2,3,4$, cf. Appendix A,

$$
\sup _{n \geq 1} \kappa_{k}\left(\bar{X}_{0}^{n}\right)=\sup _{n \geq 1} \kappa_{k}\left(M^{\prime}\right) \int_{C_{n}(0)} f(s)^{k} \mathrm{~d} s \leq \kappa_{k}\left(M^{\prime}\right) \int_{\mathbb{R}^{d}} f(s)^{k} \mathrm{~d} s<\infty .
$$

Thus also $\sup _{n \geq 1} \mathbb{E}\left(\bar{X}_{0}^{n}\right)^{4}<\infty$. By stationarity, also $\sup _{n \geq 1} \mathbb{E}\left(\bar{X}_{h}^{n}\right)^{4}<\infty$, and thus $\mathbb{E} Z^{2}<\infty$, due to Corollary B.2. Then

$$
\begin{aligned}
L_{n}(\epsilon) & =m_{n}^{2 d} \sum_{j \in \Gamma_{n}} \mathbb{E}\left[V_{n}^{2}(j) \mathbf{1}_{\left\{\left|V_{n}(j)\right| \geq \epsilon m_{n}^{-2 d}\right\}}\right] \\
& =m_{n}^{2 d} \mathbb{E}\left[\left(\left(\bar{X}_{0}^{n}-\bar{X}_{h}^{n}\right)^{2}-\gamma(h)_{n}\right)^{2} \mathbf{1}_{\left\{\left|\left(\bar{X}_{0}^{n}-\bar{X}_{h}^{n}\right)^{2}-\gamma(h)_{n}\right| \geq \epsilon \sqrt{\left|\Gamma_{n}\right|} m_{n}^{-2 d}\right\}}\right] \\
& \leq m_{n}^{2 d} \mathbb{E}\left[Z^{2} \mathbf{1}_{\left\{Z \geq \epsilon \sqrt{\left|\Gamma_{n}\right|} m_{n}^{-2 d}\right\}}\right] .
\end{aligned}
$$

Now, it follows that $L_{n}(\epsilon) \rightarrow 0$ by choosing $\left(m_{n}\right)_{n \geq 1}$ as in the proof of Theorem 2.1 for $S_{\Gamma_{n}}$. Thus, we can conclude from Theorem 3.1

$$
\frac{\bar{T}_{\Gamma_{n}}-\left|\Gamma_{n}\right| \gamma(h)_{n}}{\sqrt{\left|\Gamma_{n}\right|}} \stackrel{\mathcal{D}}{\rightarrow} \mathcal{N}\left(0, \sigma_{T}^{2}\right),
$$

and combining this with (3.4) gives the desired convergence. 


\section{Isotropic model}

Now make the assumption that the observations in the field have the form

$$
X_{i}=\int_{\mathbb{R}^{d}} f(\|s-i\|) M(\mathrm{~d} s)
$$

for $i \in \mathbb{Z}^{d}$, where $f:[0, \infty) \rightarrow[0, \infty)$ satisfies

$$
\int_{\mathbb{R}^{d}} f(\|s\|) \mathrm{d} s<\infty .
$$

This is equivalent to assuming that $f(s)$ in (2.4) only depends on $s$ through $\|s\|$. With this assumption, the field is isotropic, so

$$
\mathbb{E}\left(X_{i}-X_{j}\right)^{2}=\gamma(\|i-j\|)
$$

for a variogram function $\gamma:[0, \infty) \rightarrow[0, \infty)$. Estimation of $\mu=\mathbb{E} X_{0}$ and the corresponding asymptotic properties are unchanged, compared to the more general framework in Section 2. Also estimation of $\gamma(\|h\|)$ for $h \in \mathbb{Z}^{d}$ can be carried out as before, and the asymptotic results are still valid.

However, we can improve the variogram estimator by taking into account that $\gamma(\|h\|)$ only depends on $\|h\|$. For this, we introduce

$$
T_{\Gamma}^{\prime}\left(d_{0}\right)=\frac{1}{\left|h\left(d_{0}\right)\right|} \sum_{i \in \Gamma} \sum_{h \in h\left(d_{0}\right)}\left(X_{i}-X_{i+h}\right)^{2},
$$

where $\Gamma$ is a finite subset of $\mathbb{Z}^{d}, d_{0}>0$ and $h\left(d_{0}\right)=\left\{h \in \mathbb{Z}^{d}:\|h\|=d_{0}\right\}$. We have

Theorem 4.1. Let $\left(\Gamma_{n}\right)_{n \geq 1}$ be a sequence of finite subsets of $\mathbb{Z}^{d}$ and assume that $\left|\Gamma_{n}\right|$ goes to infinity and $\left|\partial \Gamma_{n}\right| /\left|\Gamma_{n}\right|$ goes to 0 . If

$$
\sum_{i \in \mathbb{Z}^{d}} \int_{\mathbb{R}^{d}} f(\|s\|) f(\|s-i\|) \mathrm{d} s<\infty
$$

and $\mathbb{E}\left(M^{\prime}\right)^{4}<\infty$, then

$$
\frac{T_{\Gamma_{n}}^{\prime}\left(d_{0}\right)-\left|\Gamma_{n}\right| \gamma\left(d_{0}\right)}{\sqrt{\left|\Gamma_{n}\right|}} \stackrel{\mathcal{D}}{\rightarrow} \mathcal{N}\left(0, \sigma_{T^{\prime}}^{2}\right),
$$

where

$$
\begin{aligned}
\sigma_{T^{\prime}}^{2}=\frac{1}{\left|h\left(d_{0}\right)\right|^{2}} \sum_{j \in \mathbb{Z}^{d}} \sum_{h, h^{\prime} \in h\left(d_{0}\right)}[ & \kappa_{4}\left(M^{\prime}\right) \int_{\mathbb{R}^{d}} g_{h}(s)^{2} g_{h^{\prime}}(s-j)^{2} \mathrm{~d} s \\
& \left.+2 \operatorname{Var}\left(M^{\prime}\right)^{2}\left(\int_{\mathbb{R}^{d}} g_{h}(s) g_{h^{\prime}}(s-j) \mathrm{d} s\right)^{2}\right]<\infty,
\end{aligned}
$$

and $g_{h}(s)=f(\|s\|)-f(\|s-h\|)$.

The proof of Theorem 4.1 follows exactly the same lines as the second part of the proof of Theorem 2.1. Additional notation is only needed to take care of the extra summation in the definition of $T_{\Gamma}^{\prime}$. 


\section{Estimation of sample spacing}

Assume that $d=3$ and consider the isotropic case again. Let the random field $\left(X_{t}\right)_{t \in \mathbb{R}^{3}}$ be defined as

$$
X_{t}=\int_{\mathbb{R}^{3}} f(\|s-t\|) M(\mathrm{~d} s) .
$$

Suppose that the field is only observed in grid points within two parallel planes with an unknown distance that we want to estimate. Due to the isotropy, we can without loss of generality assume that observations are made in the two planes

$$
\mathbb{Z}^{2}=\left\{\left(z_{1}, z_{2}, 0\right): z_{1}, z_{2} \in \mathbb{Z}\right\}
$$

and

$$
\mathbb{Z}^{2}+h_{0}=\left\{\left(z_{1}, z_{2}, d_{0}\right): z_{1}, z_{2} \in \mathbb{Z}\right\}
$$

where $h_{0}=\left(0,0, d_{0}\right)$. The aim is then to estimate $d_{0}$. Note that $\mathbb{Z}^{2}+h_{0} \subset \mathbb{Z}^{3}$ only if $d_{0} \in \mathbb{Z}$, which is not assumed. The idea will be to estimate $\gamma\left(d_{0}\right)$, the variogram evaluated in $d_{0}$, only based on pairs $\left(i, i+h_{0}\right)$, where $i \in \mathbb{Z}^{2}$ and $i+h_{0} \in \mathbb{Z}^{2}+h_{0}$. For a finite subset $\Gamma \subset \mathbb{Z}^{2}$, we can estimate $\gamma\left(d_{0}\right)$ by $\widehat{\gamma\left(d_{0}\right)}=\frac{1}{|\Gamma|} \tilde{T}_{\Gamma}$, where

$$
\tilde{T}_{\Gamma}=\sum_{i \in \Gamma}\left(X_{i}-X_{i+h_{0}}\right)^{2}
$$

Assuming that the variogram function $d \mapsto \gamma(d)$ is known and strictly increasing, an estimator for $d_{0}$ is obtained by

$$
\hat{d}_{0}=\gamma^{-1}\left(\widehat{\gamma\left(d_{0}\right)}\right)
$$

This estimator is studied in [13], where an expression for the variance of $\tilde{T}_{\Gamma}$ is derived. Furthermore an approximate expression for the variance of $\hat{d}_{0}$ is found using a Taylor approximation.

Below we state a theorem, giving asymptotic normality of $\tilde{T}_{\Gamma}$. In fact, apart from the scaling, the asymptotic variance is equal to the approximation to the variance suggested in [13].

A result showing asymptotic normality of $\tilde{T}_{\Gamma}$ can directly be translated into asymptotic confidence intervals for $\hat{d}_{0}$. If also $d \mapsto \gamma(d)$ is assumed to be differentiable, asymptotic normality of $\hat{d}_{0}$ is easily derived using the delta method.

Theorem 5.1. Let $\left(\Gamma_{n}\right)_{n \geq 1}$ be a sequence of finite subsets of $\mathbb{Z}^{2}$ and assume that $\left|\Gamma_{n}\right|$ goes to infinity and that $\left|\partial \Gamma_{n}\right| /\left|\Gamma_{n}\right| \rightarrow 0$ for all $j \in \mathbb{Z}^{2}$. Assume furthermore that $\mathbb{E}\left(M^{\prime}\right)^{4}<\infty$ and

$$
\sum_{i \in \mathbb{Z}^{2}} \int_{\mathbb{R}^{3}} f(\|s\|) f(\|s-i\|) \mathrm{d} s<\infty .
$$

Then,

$$
\frac{\tilde{T}_{\Gamma_{n}}-\left|\Gamma_{n}\right| \gamma\left(d_{0}\right)}{\sqrt{\left|\Gamma_{n}\right|}} \stackrel{\mathcal{D}}{\rightarrow} \mathcal{N}\left(0, \sigma_{\tilde{T}}^{2}\right),
$$


where

$$
\begin{aligned}
\sigma_{\tilde{T}}^{2}=\sum_{j \in \mathbb{Z}^{2}}[ & \kappa_{4}\left(M^{\prime}\right) \int_{\mathbb{R}^{d}} g(s)^{2} g(s-j)^{2} \mathrm{~d} s \\
& \left.+2 \operatorname{Var}\left(M^{\prime}\right)^{2}\left(\int_{\mathbb{R}^{d}} g(s) g(s-j) \mathrm{d} s\right)^{2}\right]<\infty
\end{aligned}
$$

and $g(s)=f(\|s\|)-f\left(\left\|s-h_{0}\right\|\right)$.

Above $\partial \Gamma_{n}$ denotes the boundary of $\Gamma_{n}$ as a subset of $\mathbb{Z}^{2}$. The proof of Theorem 5.1 is almost identical to the proof of Theorem 2.1 , but with $\mathbb{Z}^{d}$ replaced by $\mathbb{Z}^{2}$ as the index set for the observations.

\section{Acknowledgements}

This work was supported by Centre for Stochastic Geometry and Advanced Bioimaging, funded by the Villum Foundation.

\section{A Cumulant formulas}

For two variables $Y_{1}, Y_{2}$ on the form (2.4),

$$
Y_{1}=\int_{\mathbb{R}^{d}} f_{1}(s) M(\mathrm{~d} s) \quad Y_{2}=\int_{\mathbb{R}^{d}} f_{2}(s) M(\mathrm{~d} s),
$$

with kernels $f_{1}, f_{2}$ satisfying (2.3), the joint cumulant function is given as

$$
\log \mathbb{E}\left(e^{i\left(\lambda_{1} Y_{1}+\lambda_{2} Y_{2}\right)}\right)=\int_{\mathbb{R}^{d}} \log \mathbb{E}\left(e^{i\left(\lambda_{1} f_{1}(s)+\lambda_{2} f_{2}(s)\right) M^{\prime}}\right) \mathrm{d} s,
$$

see [6] for details. Assuming that $E\left|M^{\prime}\right|^{k}<\infty$ and differentiating $k$ times with respect to $\lambda_{1}$ gives

$$
\kappa_{k}\left(Y_{1}\right)=\kappa_{k}\left(M^{\prime}\right) \int_{\mathbb{R}^{d}} f_{1}(s)^{k} \mathrm{~d} s,
$$

where $\kappa_{k}(X)$ denotes the $k$ th cumulant of the variable $X$. Similarly, if $E\left(M^{\prime}\right)^{2}<\infty$ we find

$$
\operatorname{Cov}\left(Y_{1}, Y_{2}\right)=\operatorname{Var}\left(M^{\prime}\right) \int_{\mathbb{R}^{d}} f_{1}(s) f_{2}(s) \mathrm{d} s .
$$

If furthermore $\mathbb{E} Y_{1}=\mathbb{E} Y_{2}=0$ and $E\left(M^{\prime}\right)^{4}<\infty$, it holds

$$
\begin{aligned}
& \mathbb{E}\left(\left(Y_{1}^{2}-\mathbb{E} Y_{1}^{2}\right)\left(Y_{2}^{2}-\mathbb{E} Y_{2}^{2}\right)\right) \\
& \quad=\kappa_{4}\left(M^{\prime}\right)\left(\int_{\mathbb{R}^{d}} f_{1}(s)^{2} f_{2}(s)^{2} \mathrm{~d} s\right)+2 \operatorname{Var}\left(M^{\prime}\right)^{2}\left(\int_{\mathbb{R}^{d}} f_{1}(s) f_{2}(s) \mathrm{d} s\right)^{2} .
\end{aligned}
$$




\section{B Corollary to Ottaviani's inequality}

In this Appendix $\left(X_{n}\right)_{n \geq 1}$ will denote a sequence of random variables. Let

$$
S_{n}=X_{1}+\cdots+X_{n} \quad \text { and } \quad M_{n}=\max _{1 \leq k \leq n}\left|S_{k}\right| .
$$

We will use the following version of Ottaviani's inequality, see [7, Section 6.24].

Theorem B.1. Let $\left(X_{n}\right)_{n \geq 1}$ be a sequence of independent random variables. Then,

$$
\mathbb{P}\left(M_{n}>x+y\right) \cdot \min _{1 \leq k \leq n} \mathbb{P}\left(\left|S_{n}-S_{k}\right| \leq y\right) \leq \mathbb{P}\left(\left|S_{n}\right|>x\right)
$$

for all $x, y \in \mathbb{R}$.

Corollary B.2. Let $\left(X_{n}\right)_{n \geq 1}$ be a sequence of independent random variables. Then for all $p \geq 1, \sup _{n \geq 1} \mathbb{E}\left|S_{n}\right|^{p}<\infty$ if and only if $\mathbb{E} M^{p}<\infty$, where $M=\sup _{n \geq 1} M_{n}$.

Note that the corollary can be shown for $0<p<1$ as well. However, in the present paper we only need the result for $p=2,4$.

Proof of the corollary. First notice that it is easy to show directly that $\mathbb{E} M^{p}<\infty$ implies $\sup _{n \geq 1} \mathbb{E}\left|S_{n}\right|^{p}<\infty$. The opposite statement follows from monotone convergence, if we can show that for all $n \geq 1$

$$
\mathbb{E} M_{n}^{p} \leq C \max _{1 \leq k \leq n} \mathbb{E}\left|S_{k}\right|^{p}
$$

for a finite constant $C$ that only depends on $p$. By assumption, $\sup _{n \geq 1} \mathbb{E}\left|S_{n}\right|^{p}<\infty$ and therefore,

$$
m=\max _{1 \leq k \leq n} \mathbb{E}\left|S_{k}\right|^{p}<\infty .
$$

Let $\tau=\left(2^{p+1} m\right)^{1 / p}$. Then, by Markov's inequality, we have for $1 \leq k \leq n$

$$
\mathbb{P}\left(\left|S_{n}-S_{k}\right|>\tau\right) \leq \frac{\mathbb{E}\left(\left|S_{n}-S_{k}\right|^{p}\right)}{\tau^{p}} \leq 2^{p} \frac{\mathbb{E}\left|S_{n}\right|^{p}+\mathbb{E}\left|S_{k}\right|^{p}}{\tau^{p}} \leq \frac{1}{2}
$$

where the inequality $|x+y|^{p} \leq 2^{p}\left(|x|^{p}+|y|^{p}\right)$ has been applied. It follows that $\min _{1 \leq k \leq n} \mathbb{P}\left(\left|S_{n}-S_{k}\right| \leq \tau\right) \geq \frac{1}{2}$, so by Theorem B.1,

$$
\mathbb{P}\left(M_{n}>x\right)=\mathbb{P}\left(M_{n}>(x-\tau)+\tau\right) \leq 2 \mathbb{P}\left(\left|S_{n}\right|+\tau>x\right) .
$$

Integrating both sides with respect to $p x^{p-1} \mathrm{~d} x$ gives

$$
\mathbb{E} M_{n}^{p} \leq 2 \mathbb{E}\left(\left(\left|S_{n}\right|+\tau\right)^{p}\right) \leq 2^{p+1} \mathbb{E}\left(\left|S_{n}\right|^{p}+\tau^{p}\right) \leq 2^{p+1}\left(1+2^{p+1}\right) m .
$$




\section{References}

[1] Adler, R. J., Samorodnitsky, G. and Taylor, J. E. (2010). Excursion sets of three classes of stable random fields. Adv. Appl. Prob. 42, 293-318.

[2] Adler, R. J., Samorodnitsky, G. and Taylor, J. E. (2013). High level excursion set geometry for non-Gaussian infinitely divisible random fields. Ann. Prob. 41, 134-169.

[3] Barndorff-Nielsen, O. E. And Schmiegel, J (2004). Lévy based tempospatial modelling with applications to turbulence. Uspekhi Mat. Nauk. 159, 63-90.

[4] El Machkouri, M., Volný, D. and Wu, W. B. (2013). A central limit theorem for stationary random fields. Stoch. Proc. Appl. 123, 1-14.

[5] HeinRICH, L. (1988). Asymptotic behaviour of an empirical nearest-neighbour distance function for stationary Poisson cluster processes. Math. Nachr. 136, $131-148$.

[6] Hellmund, G., Prokešová, M. and Jensen, E. B. V. (2008). Lévy based Cox point processes. Adv. Appl. Prob. 40, 603-629.

[7] Hoffmann-Jørgensen, J. (1994). Probability with a View towards Statistics. Vol. I. Chapmann \& Hall, New York and London.

[8] Jónsdóttir, K. Ý., Schmiegel, J. And Jensen, E. B. V. (2008). Lévybased growth models. Bernoulli 14, 62-90.

[9] Jónsdóttir, K. Ý., Rønn-Nielsen, A., Mouridsen, K. And Jensen, E. B. V. (2013). Lévy-based modelling in brain imaging. Scand. J. Stat. 40, $511-529$.

[10] Rajput, B. S. And Rosiński, J. (1989). Spectral representations of infinitely divisible processes. Prob. Theory Rel. Fields 82, 451-488.

[11] Rosiński, J. And Samorodnitsky, G. (1993). Distributions of subadditive functionals of sample paths of infinitely divisible processes. Ann. Probab. 21, 996-1014.

[12] Rønn-Nielsen, A. And Jensen, E. B. V. (2016). Tail asymptotics for the supremum of an infinitely divisible field with convolution equivalent Lévy measure. J. Appl. Prob. 53, 244-261.

[13] Rønn-Nielsen, A., Sporring, J. And Jensen, E. B. V. (2017). Estimation of sample spacing in stochastic processes. Image Anal. Stereol. 36, 43-49.

[14] Rønn-Nielsen, A. And Jensen, E. B. V. (2017). Excursion sets of infinitely divisible random fields with convolution equivalent Lévy measure. J. Appl. Prob. $\mathbf{5 4}, 833-851$. 
[15] Sato, K.-I. (1999). Lévy Processes and Infinitely Divisible Distributions. Cambridge University Press, Cambridge. 